PAPER

\section{Spatial resolution dependence on spectral frequency in human speech cortex electrocorticography}

To cite this article: Leah Muller et al 2016 J. Neural Eng. 13056013

View the article online for updates and enhancements.
Related content

Spatial co-adaptation of cortical control columns in a micro-ECoG brain-computer interface

A G Rouse, J J Williams, J J Wheeler et al.

Mapping the fine structure of cortical activity with different micro-ECoG electrode array geometries

Xi Wang, C Alexis Gkogkidis, Olga Iljina et al.

- Characterization of the effects of the human dura on macro- and microelectrocorticographic recordings David T Bundy, Erik Zellmer, Charles M Gaona et al.

\section{Recent citations}

- Sub-millimeter ECoG pitch in human
$\frac{\text { enables higher fidelity cognitive neural }}{\text { state estimation }}$
John Hermiz et al
- Cortical travelling waves: mechanisms and
$\underline{\underline{\text { computational principles }}}$
Lyle Muller et al
- Flexible Graphene Solution-Gated Field-
Effect Transistors: Efficient Transducers
for Micro-Electrocorticography
Clement Hébert et al




\title{
Spatial resolution dependence on spectral frequency in human speech cortex electrocorticography
}

\author{
Leah Muller ${ }^{1,2,3}$, Liberty S Hamilton ${ }^{1,4}$, Erik Edwards ${ }^{1,4}$, \\ Kristofer E Bouchard ${ }^{5}$ and Edward F Chang ${ }^{1,4}$ \\ ${ }^{1}$ Department of Neurological Surgery and Department of Physiology, University of California, San \\ Francisco, 675 Nelson Rising Lane, Room 511, San Francisco, CA 94158, USA \\ 2 Joint Program in Bioengineering, UC Berkeley/UC San Francisco, USA \\ ${ }^{3}$ Medical Scientist Training Program, UC San Francisco, USA \\ ${ }^{4}$ Center for Integrative Neuroscience, University of California, San Francisco, San Francisco, CA, USA \\ ${ }^{5}$ Biological Systems and Engineering Division, Lawrence Berkeley National Laboratory, Berkeley Hills, \\ CA, USA
}

E-mail: edward.chang@ucsf.edu

Received 10 November 2015, revised 24 July 2016

Accepted for publication 25 July 2016

Published 31 August 2016

\begin{abstract}
Objective. Electrocorticography $(\mathrm{ECoG})$ has become an important tool in human neuroscience and has tremendous potential for emerging applications in neural interface technology. Electrode array design parameters are outstanding issues for both research and clinical applications, and these parameters depend critically on the nature of the neural signals to be recorded. Here, we investigate the functional spatial resolution of neural signals recorded at the human cortical surface. We empirically derive spatial spread functions to quantify the shared neural activity for each frequency band of the electrocorticogram. Approach. Five subjects with high-density ( $4 \mathrm{~mm}$ center-to-center spacing) ECoG grid implants participated in speech perception and production tasks while neural activity was recorded from the speech cortex, including superior temporal gyrus, precentral gyrus, and postcentral gyrus. The cortical surface field potential was decomposed into traditional EEG frequency bands. Signal similarity between electrode pairs for each frequency band was quantified using a Pearson correlation coefficient. Main results. The correlation of neural activity between electrode pairs was inversely related to the distance between the electrodes; this relationship was used to quantify spatial falloff functions for cortical subdomains. As expected, lower frequencies remained correlated over larger distances than higher frequencies. However, both the envelope and phase of gamma and high gamma frequencies $(30-150 \mathrm{~Hz})$ are largely uncorrelated $(<90 \%)$ at $4 \mathrm{~mm}$, the smallest spacing of the high-density arrays. Thus, ECoG arrays smaller than $4 \mathrm{~mm}$ have significant promise for increasing signal resolution at high frequencies, whereas less additional gain is achieved for lower frequencies. Significance. Our findings quantitatively demonstrate the dependence of ECoG spatial resolution on the neural frequency of interest. We demonstrate that this relationship is consistent across patients and across cortical areas during activity.
\end{abstract}

Keywords: electrocorticography, spatial, resolution, cortical surface, optimal spacing, ECoG array, human

(Some figures may appear in colour only in the online journal) 


\section{Introduction}

Electrocorticography $(\mathrm{ECoG})$ is a method of recording electrical neural signals from the cortical surface and is a wellestablished clinical tool for localizing seizure foci in epileptic patients. Clinical ECoG records neural activity using electrodes positioned in a grid arrangement, usually spaced $1 \mathrm{~cm}$ apart. More recently ECoG has found utility in both scientific and engineering applications due to its high spatial and temporal resolution relative to noninvasive imaging approaches. In research applications, high frequency neural responses have been shown to be informative for task-related neural decoding, while low frequency bands can serve as markers for neural states (Fries et al 2007, Miller et al 2007, Womelsdorf and Fries 2007, Ray et al 2008, Engel and Fries 2010, Kellis et al 2010, Crone et al 2011, Lachaux et al 2012). Moreover, high frequency neural signals have been described to be more spatially discrete than low frequency signals (Buzsaki and Draguhn 2004, Edwards et al 2009, Pesaran 2010, Viventi et al 2011, Leski et al 2013). However, the spatial resolution of neural signals from the human cortical surface with respect to these functional neural frequency bands has never been fully quantified in humans.

Higher density ECoG grids collect more information from the cortical surface than the standard $1 \mathrm{~cm}$ grids, leading to better decoding performance in brain machine interface applications (Blakely et al 2008, Leuthardt et al 2009, Wang et al 2009, Kellis et al 2010, Breshears et al 2011, Flinker et al 2011, Khodagholy et al 2015). In most cases, these high density arrays have covered a very small area of cortex, and therefore large scale comparisons of shared signal in space have not been evaluated. Recently, correlations in the raw signal have been explored with respect to distance using a clinical ECoG array and a microwire ECoG array (Kellis et al 2015). However, the measured signals from the microwire array are inherently different from those of the clinical array due to filtering effects of electrode size and shape (Lempka et al 2011, Kent and Grill 2014). Furthermore, the microwire array can examine signal similarity only within a very small area of cortex, while the clinical array sparsely samples a wide expanse of cortex that may or may not be actively engaged at the time of recording.

Ultra high-density, low impedance electrode arrays for chronic human recordings are not presently FDA approved in the United States, which is a dilemma for empirically deriving spatial resolution values for this work. Work in animal models can give us some insight into spatial correlations at higher electrode density (Katzner et al 2009, Xing et al 2009, Kajikawa and Schroeder 2011); however, it may be that spatial correlations in human cortex differ due to differences in underlying cytoarchitecture, cortical thickness, and differences in cortical folding across species (Elston 2003). Thus, we hope that this study serves to illustrate the utility of moving toward higher-density arrays in human subjects as well. A high-density ECoG grid with $4 \mathrm{~mm}$ spacing of electrodes offers a unique opportunity to identify shared signal at close spacing of electrodes, over a broad area of active cortex, and across neural frequency bands. We describe frequency band-specific spatial spread at the cortical surface in patients performing speech-related tasks. We use these observations to compare spatial spread functions across patients and across functional areas during speech perception and production tasks.

\section{Materials and methods}

\subsection{Experimental setup}

2.1.1. Subjects. Experimental procedures were approved by the Committee for Human Research at the University of California, San Francisco, and all participants provided written informed consent. Patients undergoing ECoG grid implantation for epilepsy focus localization $(N=5,0 \mathrm{M} / 5 \mathrm{~F}$, 25-41 years old) volunteered to participate in this research study. Patients were implanted with 256 channel high-density subdural electrode arrays (AdTech, Racine, WI or Integra, Plainsboro, NJ) with $4 \mathrm{~mm}$ center-to-center spacing $(1.17 \mathrm{~mm}$ exposed diameter). Electrodes were positioned according to clinical criteria but included coverage over the superior temporal gyrus (STG) and the ventral sensorimotor cortex (pre- and post-central gyri). An electrode in the sub-galeal space served as the reference electrode and ground.

2.1.2. Tasks and stimuli. The patients either spoke or listened to sentences taken from the TIMIT database (Garofolo 1993, Wrench 1999). In the listening task, patients heard a subset of 125 sentences drawn pseudo-randomly from a list of 499 sentences spoken by 354 male and 145 female speakers. In the speaking task, patients were instructed to read a set of 50 sentences, presented visually on a laptop screen at a userdriven pace. In both tasks, sentences were chosen to evenly sample the distribution of phonemes present in English. These tasks lasted approximately 5-10 min each. Presentation of experimental stimuli was synchronized to neural recordings and controlled using custom MATLAB software running on a Windows laptop.

\subsection{Recording and data processing}

Signals were acquired at a sampling rate of $3051.8 \mathrm{~Hz}$ and were amplified and digitized using a Tucker-Davis Technologies (Alachua, FL, USA) neural recording system (RZ2 DSP combined with a 256-channel PZ2 amplifier). Manual artifact and channel rejection was performed, followed by common average referencing (CAR) (Crone et al 2001). The CAR consists of the mean across sixteen-channel banks of non-rejected electrodes that are spatially arranged in a row that spans $6 \mathrm{~cm}$. The CAR approximates the activity contributed by the original reference, so subtracting the CAR from each channel largely removes contributions of the original reference electrode and electrical artifacts that are often present in groups of sixteen channels due to shared connectors and cabling (see Crone et al 2001 for a discussion of CAR in ECoG recordings). The CAR can also be thought of 
as the spatial DC portion of the signal, and CAR-re-referencing as a typical mean or DC removal.

The signal from each electrode channel was filtered into 40 frequency bands using an FFT followed by a Hilbert transform with logarithmically increasing center frequencies (range of CF $=4.07-193.8 \mathrm{~Hz}$ ). The analytic amplitude, also known as the envelope, and the phase were calculated for each (Edwards et al 2009). The filtered frequencies were then averaged into frequency bands corresponding to the traditional theta $(4-7 \mathrm{~Hz})$, alpha $(7-14 \mathrm{~Hz})$, beta $(15-30 \mathrm{~Hz})$, gamma $(30-60 \mathrm{~Hz})$, and high gamma $(70-150 \mathrm{~Hz})$ bands. This signal was then $z$-scored with respect to rest (no-task) epochs within the same recording. The anatomical location of electrodes was determined with the aid of co-registered brain CT and MRI images. The precentral gyrus (preCG), the postcentral gyrus (postCG), the suprasylvian region, the STG, and the middle temporal gyrus (MTG) were grouped and evaluated separately for some analyzes.

\subsection{Analytical methods}

Electrodes were chosen for analysis within an anatomically defined area using a bootstrap t-test to find electrodes that responded significantly to speech over silence in the high gamma band. The high gamma band was chosen because it has been shown to exhibit specificity in activation to speech sounds in eloquent cortex. Electrode responses during speech were compared to responses during silence using short consonant-vowel syllables as the stimulus. The threshold $p$-value for inclusion was $p<0.001$ over 10000 bootstrap iterations. Of the 256 electrodes on the grid, an average of $80 \pm 6$ electrodes were anatomically localized to the STG, which is expected to respond to speech sounds. Of these anatomically localized electrodes, an average of $87 \%$ showed significant responses to speech over silence.

A Pearson correlation coefficient was calculated between the time series of either the analytic amplitude or the phase of each electrode pair within the anatomically and functionally defined subset (described above) and associated with the Euclidean distance between the electrodes. Figure 1 shows representative data along with its subsequent comparison for two nearby electrodes. For simplicity, the term 'component' will be used to refer to either the analytic amplitude or the phase of a single frequency band for a single patient. The entire 5-10 min of data from each task were used to derive the correlation coefficient for the aforementioned subsets of electrode pairs. Both the electrode subset and the time series used to derive correlation coefficients varied by task. The datasets for the two tasks were analyzed independently. We calculated chance level correlations for each frequency band by randomizing the data for each component in time for each electrode separately and repeating the correlation measures for all distances. Correlations for the randomized data fell very close to zero $\left( \pm 5 \times 10^{-4}\right)$ irrespective of frequency band and distance.

To define the spatial relationship of correlation between electrode pairs, we modeled the falloff of the electrode correlations versus distance, using the interquartile range
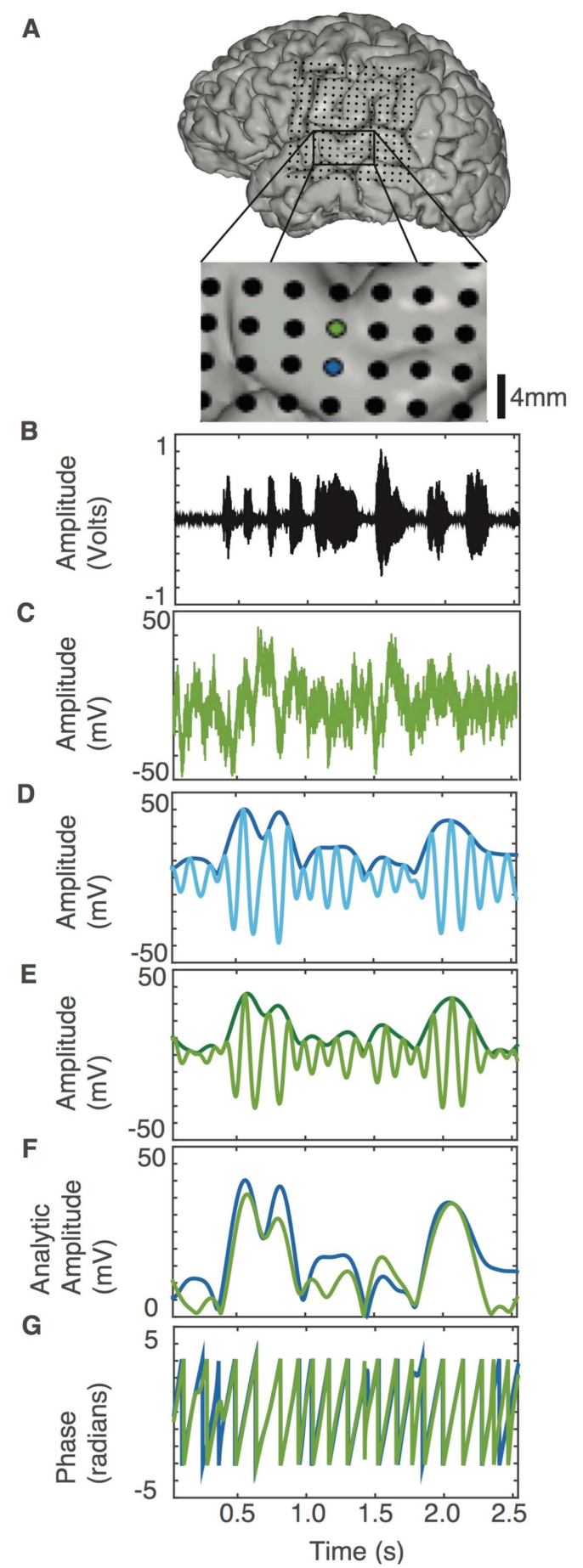

Figure 1. Comparing ECoG signals from adjacent electrodes. (A) MRI reconstruction with CT co-registered electrode positions. (Inset) Two adjacent electrodes are spaced $4 \mathrm{~mm}$ apart on the STG (green and blue). (B) Sound waveform of example sentence presented aurally. (C) Raw ECoG signal from green electrode (after notch filtering and common average referencing). (D)-(E) Timelocked neural signal from the blue (D) and the green (E) electrodes in the theta range. Light color shaded lines are bandpassed signal in the theta range, while dark colored lines are the analytic amplitude of the signal. (F) Analytic amplitudes are overlaid, and show high correlation $(R=0.83$, over approximately $5 \mathrm{~min}$ of data). (G) Phase traces for the same electrodes show high correlation $(R=0.65$, also over approximately $5 \mathrm{~min}$ of data). 
of the data for each component. We tested exponential (equation (1)), Lorentzian (equation (2)), Gaussian (equation (3)), and power law (equation (4)) functions to describe this falloff. These equations were chosen to fit the data because they are typical equations that describe nonlinear fall offs and that allow for the necessary observation that when distance is zero, correlation is one. A constant term was added where necessary to correct for the noise floor of the correlation dataset, which is most likely due to either shared activity from the reference electrode or shared noise (Bruns et al 2000). In each case, the number of free parameters was limited to two to avoid over-fitting of the function to the data.

$$
\begin{gathered}
y=(1-c) e^{-B x}+c, \\
y=(1-c) \frac{B^{2}}{B^{2}+x^{2}}+c, \\
y=(1-c) \frac{e^{-x^{2}}}{B}+c, \\
y=(x+1)^{-B} .
\end{gathered}
$$

These functions were fit separately for each component, allowing maximum goodness of fit and equal opportunity for different functions to describe the different frequencies. The best-fit equation was chosen as the one with the highest $R$ squared value using a least-squares method. For each component, the best-fit function across patients was chosen to represent the average falloff of correlation versus distance. To adequately capture the curvature of the falloff, we used only pairwise correlations at distances of $20 \mathrm{~mm}$ and below. Using data in this range minimizes the contribution to the fit from the noise floor of low correlation, which is also captured in the constant $c$. The fits with the highest $R$-squared values were corroborated by visual inspection; the data were well approximated by the chosen fit for every patient at every frequency.

\section{Results}

The goal of our study was to quantify the average spatial spread at the cortical surface for each functional frequency band. Using high-density ECoG arrays and data from human intracranial recordings, we applied a correlation-based analysis to determine the extent of shared signal across the cortical surface for the amplitude and phase content of five frequency bands.

A natural starting point for estimating spatial falloff is to look at that of the raw signal before decomposing the signal into frequency bands. This is shown in figure 2, where for each distance of separation on the electrode grid, the interquartile range of data points is plotted as a box plot. The majority of electrode pairs are correlated above noise floor only within $2 \mathrm{~cm}$; after this distance of separation between electrodes the plot approximates a horizontal line. Therefore, only electrodes located $2 \mathrm{~cm}$ or less from one another were used for analyzes. We then fit curves to the data points corresponding to electrode pair correlations to describe the falloff of correlation with distance. The best fit in this

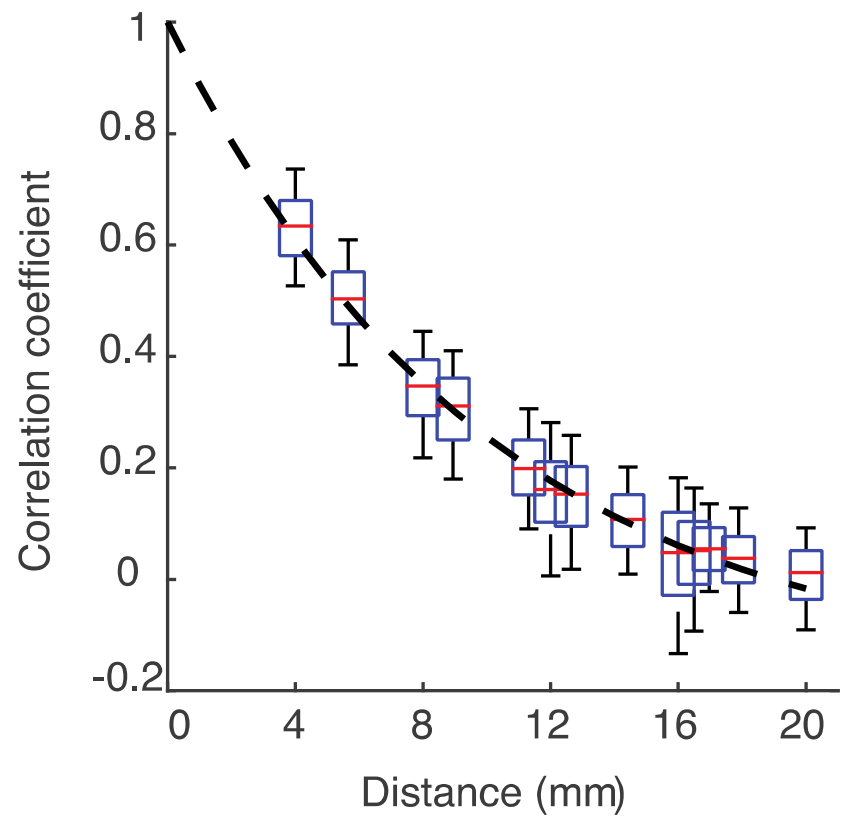

Figure 2. Raw signal correlation decreases with distance. The falloff of correlation coefficient for pairs of neural signals as distance between electrode pairs increases. Blue outline represents the interquartile range of data. Red lines are the median of each plot. Black whisker lines represent the upper and lower quartiles of data. Black dashed line shows the exponential fit, chosen as such because it has the highest $R^{2}$ value of the functions that attempted to fit the data. There are no observations below $4 \mathrm{~mm}$, the closest spacing of electrodes.

example is plotted with a dashed line. Note that the curve continues to points that are unobservable by the high-density grid, including distances less that $4 \mathrm{~mm}$. This part of the curve is extrapolated, and it can give us intuition about what correlations would be observed in the raw signal with more closely spaced electrodes, with the caveat that correlations at distances below $1.17 \mathrm{~mm}$ (the contact diameter of the electrode) are underestimated.

Correlations at low frequencies are higher in magnitude and more widespread than at high frequencies. This is shown in figure 3 for the theta and the high gamma frequency bands. The high gamma band signals at nearby electrodes show a lower maximum correlation in comparison to the theta band signals, and the spatial spread of notable correlations encompasses less area on the cortical surface than that of theta. As expected, we observe a decrease in correlation between pairs of electrodes as the distance increases between them.

The correlations between electrode pairs differ predictably not only by frequency band but also by whether or not the patient was engaged in a task. The tasks that we examined were speech perception and production. The rest versus task distinction is particularly evident in the high gamma band for electrodes that are considered active (see methods for details in determining which are active). Figure 4 shows the drastic difference in correlation patterns with distance for electrodes in the STG when the patient listened to speech versus when the patient sat quietly. Points at each 

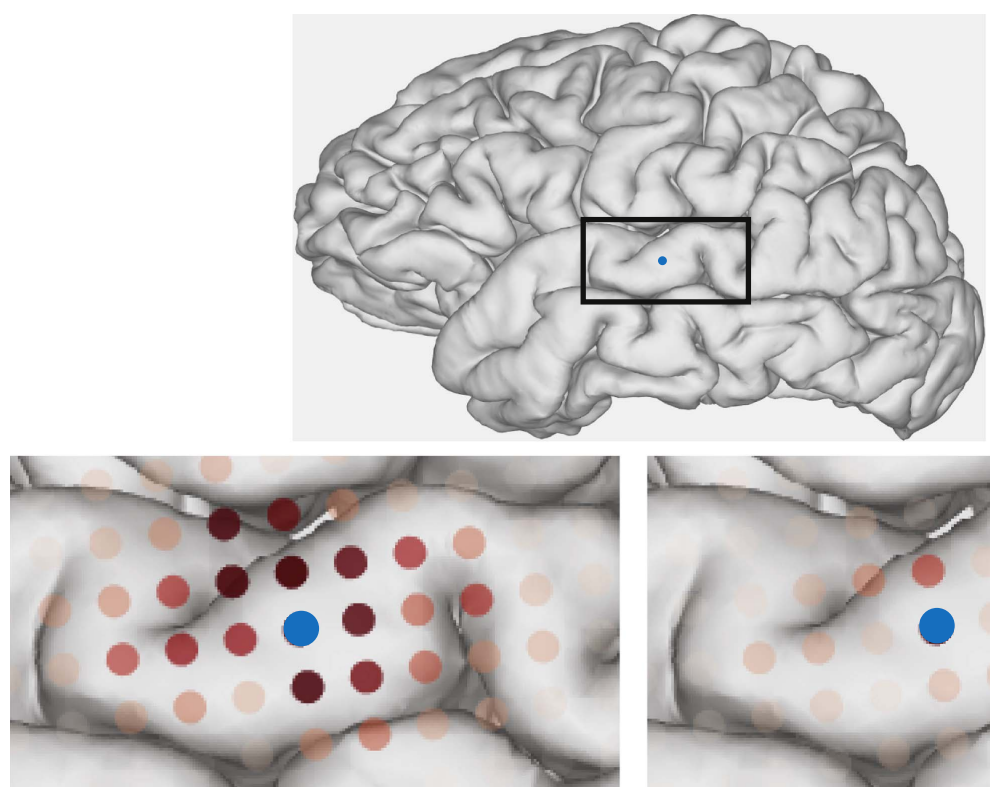

Theta

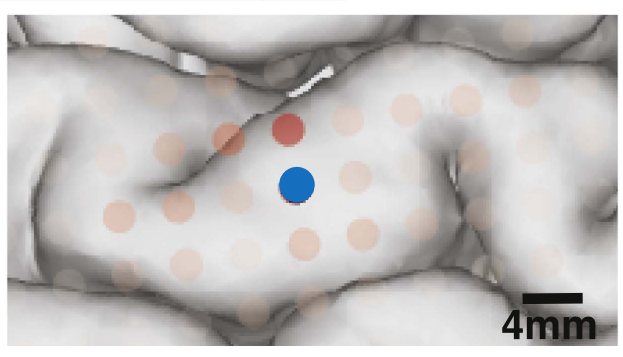

High gamma
0.5

0.45

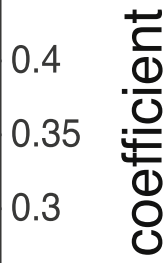

0.25

$-0.2$

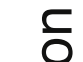

$-0.15$

0.1

0.05

0

Figure 3. Comparison of ECoG spatial spread for low and high spectral frequency bands. The part of the brain that is represented in the insets is shown in the boxed portion of the full brain above. In the inset to the left, correlations in analytic amplitude for the theta band are shown for one electrode. The same electrode is shown at right, along with correlations in the analytic amplitude of the high gamma frequency band. The theta band (left) shows higher correlations over a larger area than the high gamma band (right). The electrode to which all pairwise correlations are made is shown in blue for each.

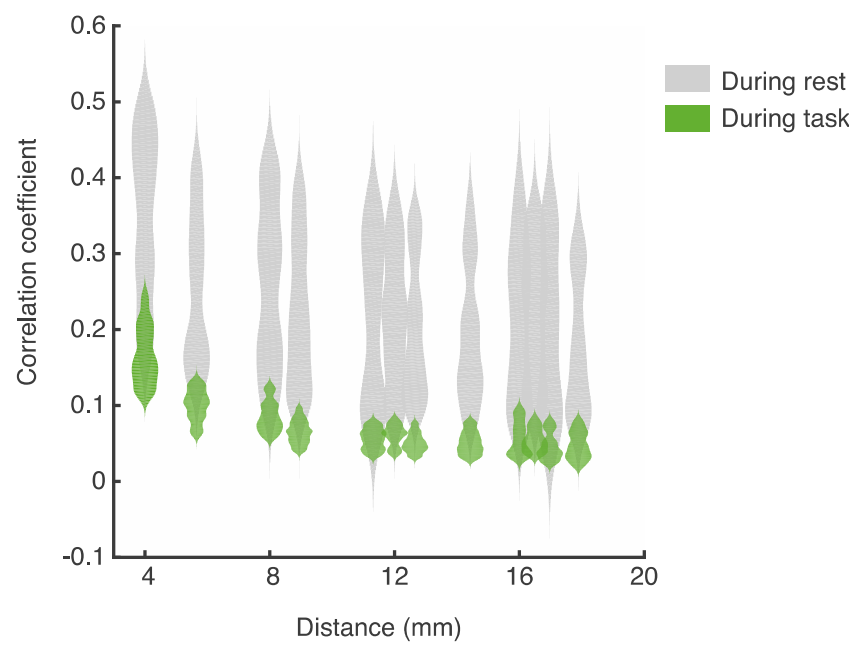

Figure 4. Distributions of high gamma correlations for rest versus activity. Correlations decrease markedly in the high gamma frequency when the subject performs a task. Distributions at each distance are shown for the high gamma analytic amplitude activity for active electrodes in STG of one patient during a listening task. The gray plots show the distributed values of correlations between electrodes at each distance during rest, while the green plots show this distribution during the task. At each distance, the same number of data points are used to create the distributions in gray and green.

distance are represented in a distribution plot to compare the shape of distributions. The correlations are more variable and on average higher during rest than when the patient is listening to speech. Both the median and the mean are lower during activity than during rest at all distances. A paired t-test was performed for the correlation values at each distance, and at all distances shown (distance $<20 \mathrm{~mm}$ ) there were statistically significant differences in correlations for task versus rest $(p<0.05)$. Similar differences in correlation with activity have been described elsewhere (Buckner and Vincent 2007, Cohen and Kohn 2011, Fukushima et al 2012). Because correlations decrease with activity, the spatial spread function shows a steeper fall off during activity than during rest. Considering this fact, and additionally considering that the neural signal of interest is most likely elicited while a subject is engaged in a task of some sort, we decided to base the remainder of our analyzes on electrodes that are considered active in an anatomical area, during a stimulus that elicits that activity.

As mentioned in section 2.3, four functions were fit to the data. Representative data from the theta band of one subject is shown with all fit lines superimposed in figure 5 . The Lorentzian and exponential forms were similar in shape and in goodness-of-fit, and the $R$-squared value for goodness-of-fit was quite high for both $\left(R^{2}\right.$ Lorentzian $=0.93$, $\left.R^{2}{ }_{\text {exponential }}=0.92\right)$. Indeed, the best fit choice was either Lorentzian or exponential for all components tested; however, the difference between $R^{2}$ values for these models increased for high neural frequencies (gamma and high gamma). Regardless of whether the function tested was Lorentzian or exponential, the resulting fit lines for correlation decrease with distance differed only slightly. In all cases, the Lorentzian and exponential forms were greatly superior to the Gaussian and Power Law forms in describing the falloff in correlation for each component. 


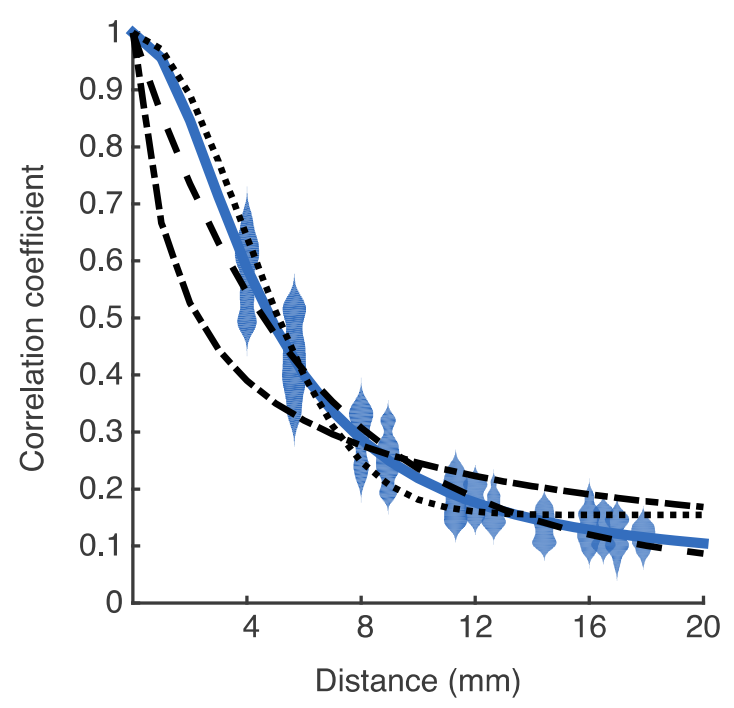

Figure 5. Best fit functions approximate representative data (theta band analytic amplitude) for a single subject. A distribution plot of pairwise correlations at each distance are shown in blue. The best fit line for this component is a Lorentzian fit, shown by the line in blue. The Lorentzian form is followed closely in goodness-of-fit by the exponential form, shown by a dashed line. The goodness-of-fit further decreases for the Gaussian fit, and finally for the Power Law.
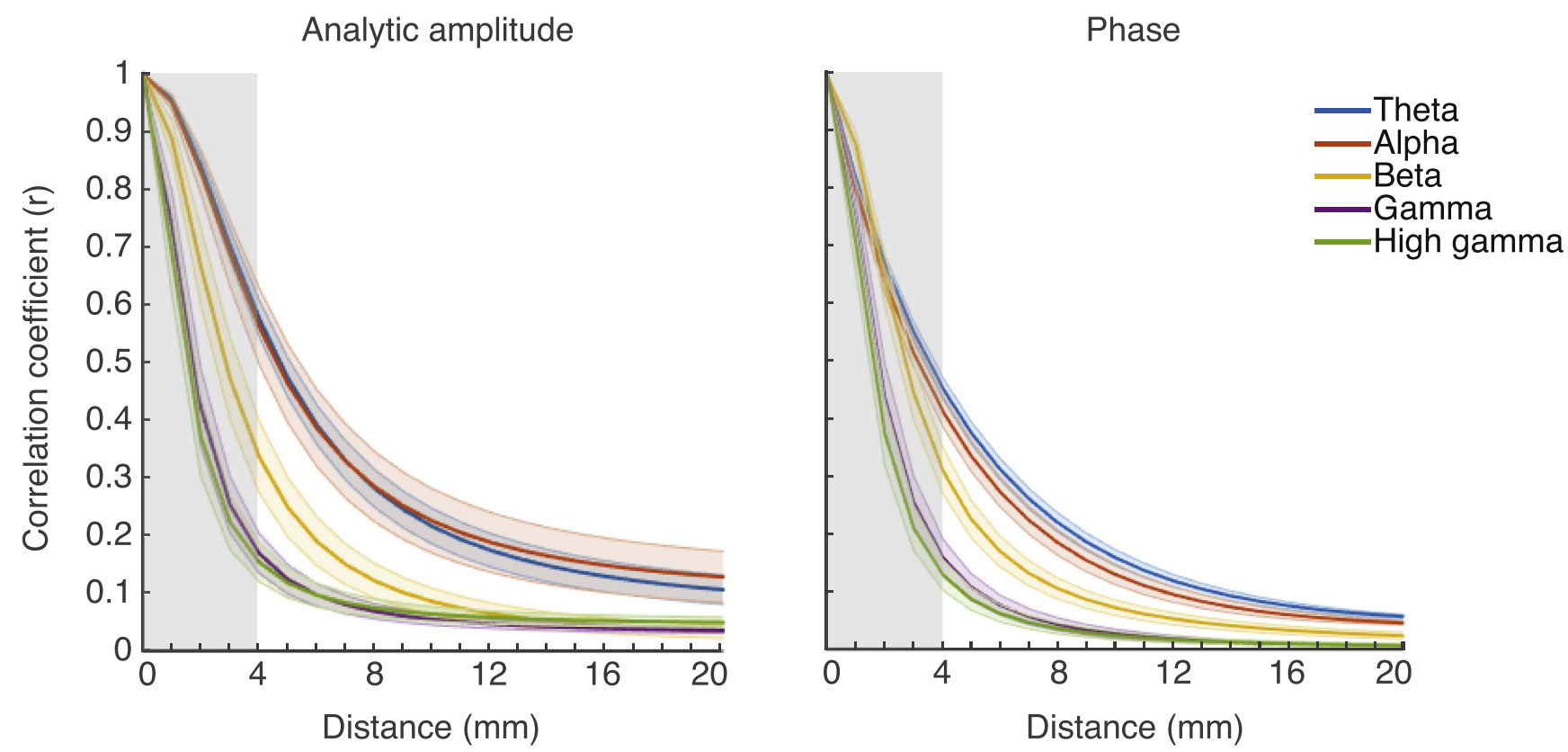

Figure 6. Electrode pair correlations decrease predictably as a function of distance. A curve-fitting algorithm was used to fit four equations to the data. The fit with the highest $R$-squared value was chosen to represent the frequency band. Fit lines become steeper with increasing neural frequency for all patients, indicating a sharper falloff of shared variability for high frequency bands with distance. The result is averaged over five patients for active electrode pairs over the STG. Dark lines indicate the mean fit across patients; lighter background fill indicates standard error across patients. The gray shaded region is the region of the fit in which there were no neighboring electrode pairs below $4 \mathrm{~mm}$.

Across frequency bands there is a nonlinear falloff of correlation with distance for both amplitude and phase of the signal. The falloff becomes steeper as the neural frequency increases. This is shown in figure 6 where best fits are averaged over five patients. The fits shown are for electrodes in the STG while the patients listened to sentences. There is a clear similarity in falloff pattern for theta and alpha bands and for gamma and high gamma bands. In the analytic amplitude data, correlation falloffs were best fit by a Lorentzian function in individual patients as well as the subject-pooled data. For phase correlations, theta and alpha were best fit by an exponential function, whereas beta, gamma, and high gamma were best fit by the Lorentzian. Importantly, the best fit function in each case was identical across patients. This did not have to be the case, since the analytic amplitude and phase for each frequency band for each patient were fit separately. The functional form of best fit for any patient could have differed from that of the other patients for the same component; 
A

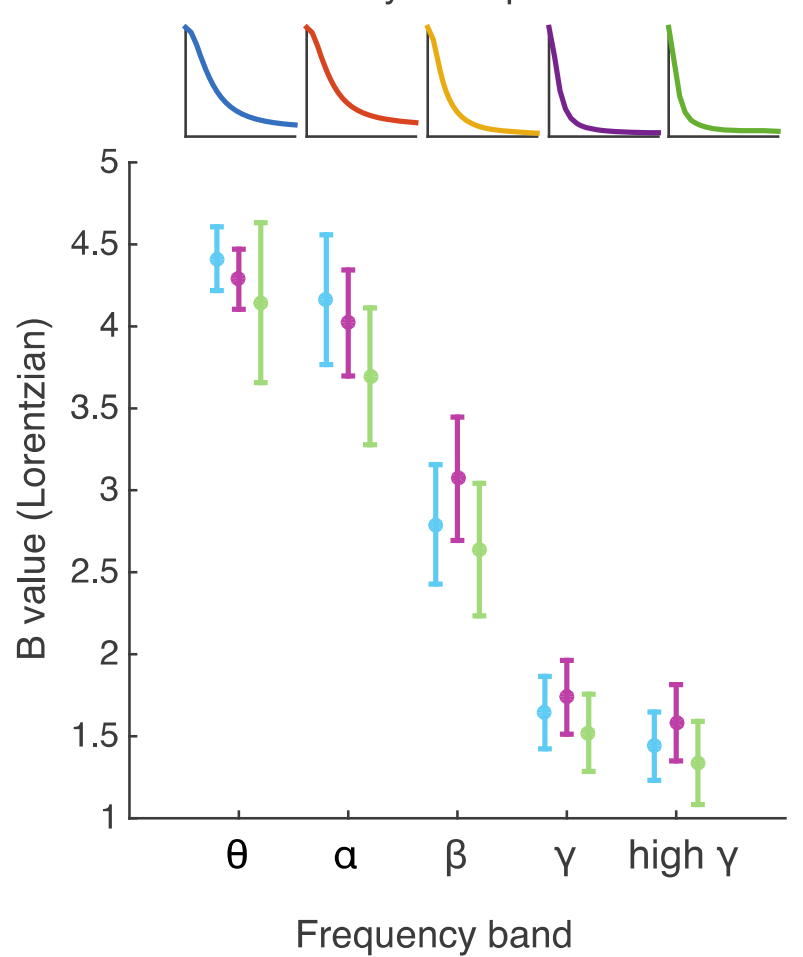

B

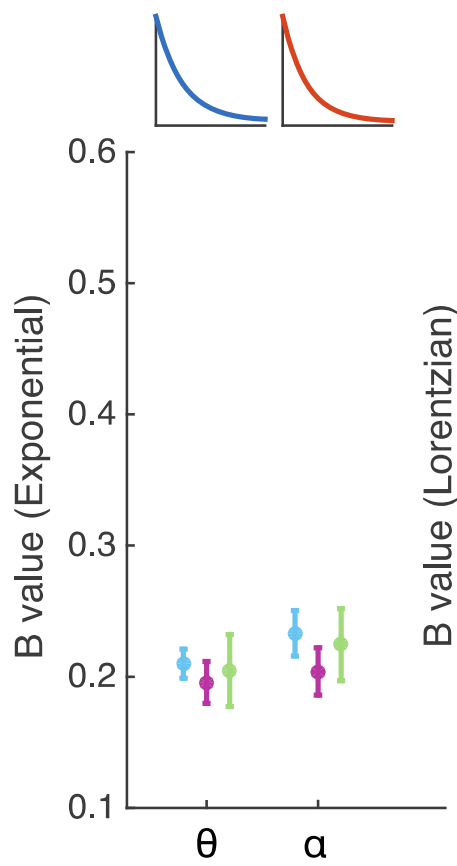

Phase

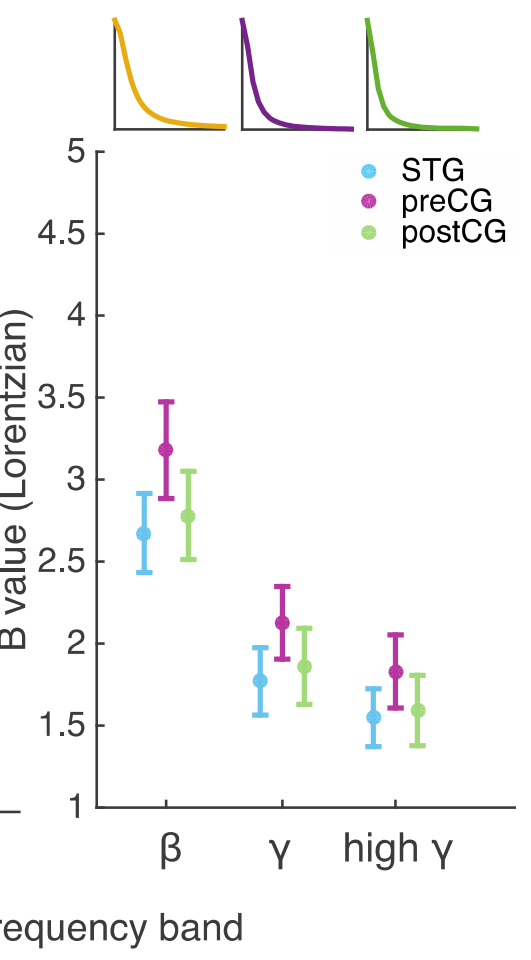

Figure 7. The steepness of correlation falloff values are conserved across patients and cortical regions, but differ by frequency band. (A) The Lorentzian equation (see methods, equation (2)) is the equation of best fit to represent falloff of correlation with distance for all frequency bands. The $B$ value is representative of the steepness of the curve. The mean and standard error of the $B$ value across patients for each cortical area is represented with a center point and error bars at each frequency band. The error bars and points are color coded by cortical area. A representative curve is shown above for each frequency band to give an idea of the curve that is constructed with the average $B$ value. (B) The exponential equation (see methods, equation (1)) is the equation of best fit to represent falloff of correlation with distance for theta and alpha frequency bands, while the Lorentzian fit best describes falloff of correlation with frequency for beta, gamma, and high gamma bands. The $B$ value is representative of the steepness of the curve. The mean and standard error of the $B$ value across patients for each cortical area is represented with a center point and error bars at each frequency band. The error bars and points are color coded by cortical area. A representative curve is shown above for each frequency band to give an idea of the curve that is constructed with the average $B$ value.

however, all components of the same class were fit best with the same functional form across all five patients. This made the task of defining a best fit curve for each component easy because we simply average the coefficients of best fit for each component across patients.

To determine if the form or slope of the best fit function differs by anatomical location, we then took correlations between electrodes overlying the preCG and postCG while the patient spoke sentences, a task that evokes reliable activity in the sensorimotor cortex (Bouchard et al 2013). The correlation analysis was repeated for these anatomical areas. Again we found the same effect: a steeper correlation falloff with increasing neural frequency. On closer comparison of fits between areas, we found that the spatial correlation falloff fit lines for preCG, postCG, and STG did not differ significantly ( $p>0.05$, paired t-test with Bonferroni correction for multiple comparisons). Therefore, the presented results also apply to the pre- and post-central gyri as well as to the STG. This is shown in figure 7 as a function of the coefficient $B$, which represents the steepness of descent of the fits. This coefficient value is conserved over patients and anatomical areas, but notably not across frequency bands. The shift in value can be noted best for the same function form for all analytic amplitude fall offs. The $B$ values shift from a relatively high value to a lower value as frequency band increases, corresponding to the increased steepness of the fall off with distance. The same pattern is seen with best fits in the phase data.

Our estimations to this point were mostly constrained to a single gyrus and by nature assume a continuous surface. We next wanted to determine how anatomical factors such as cerebral convolutions and lobar divisions affect spatial correlations. We therefore analyzed the spatial correlations between electrodes that were within a single gyrus, across a sulcus (the superior temporal sulcus between the STG and MTG), and across brain lobes (the Sylvian Fissure, between the frontal and temporal lobe). We hypothesized that spatial correlations within the STG would be significantly greater than across the superior temporal sulcus, and even more so than across the Sylvian Fissure. To test this prediction, we shifted our focus from the interquartile range to the upper quartile range of values so that high correlation values were preserved. We observed that correlations were higher between electrodes within a gyrus and that patterns of correlation seemed to cluster with anatomical boundaries such as sulci. This relationship was quantified for electrodes in the STG to electrodes in the MTG and for electrodes in the STG to 


\section{Analytic amplitude}

Phase
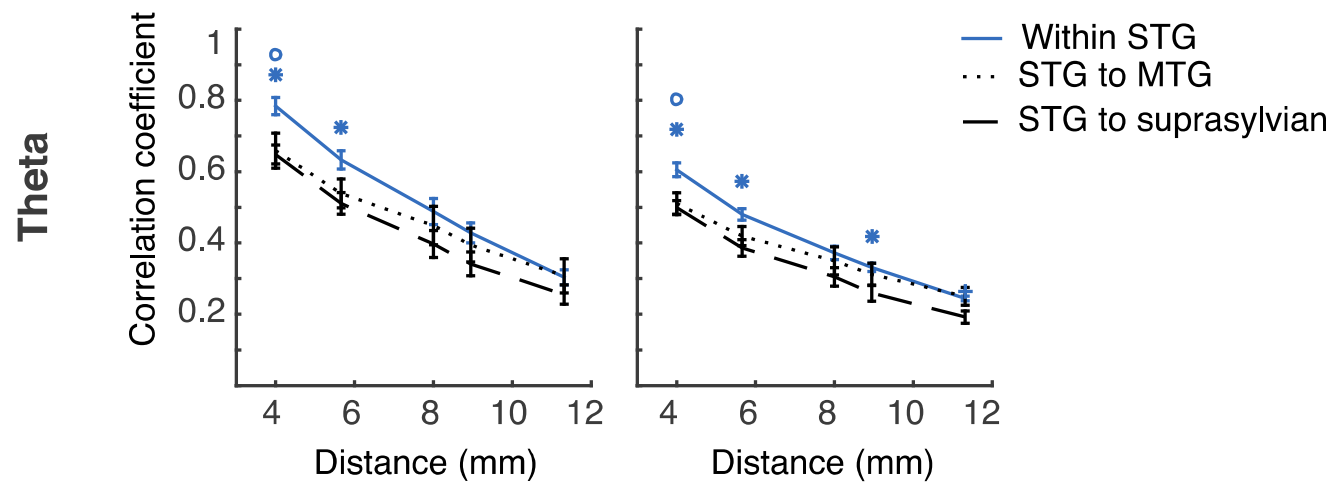

Figure 8. Within gyrus, across sulcus, and across fissure correlation patterns for the theta band. Correlations within a gyrus trend higher than correlations across a sulcus or the Sylvian fissure. Mean and standard error of correlations over patients are plotted for electrodes within the STG (blue solid line), STG to MTG (dotted line), and STG to IFG (dashed line). This effect is more pronounced for amplitude correlations than phase correlations. Statistical significance, determined with an ANOVA followed by paired t-tests at each distance, is denoted by markers above each distance. An asterisk denotes a significance of $p<0.05$ between groups 'Within STG' and 'STG to suprasylvian'. A circle denotes significance of $p<0.05$ between groups 'Within STG' and 'STG to MTG'.

electrodes across the Sylvian fissure. An ANOVA was performed between groups for each distance, followed by posthoc pairwise t-tests where applicable. The most convincing differences were in the low neural frequencies. The theta band observations are shown in figure 8. For the theta frequency band at near distances, the STG-STG electrodes were more highly correlated than the STG-MTG electrodes and the STGIFG (suprasylvian) electrodes. At distances of $5.65 \mathrm{~mm}$ (for diagonally adjacent electrodes) and $4 \mathrm{~mm}$, the STG-STG electrode correlations differ from STG-IFG correlations but do not differ significantly from the STG-MTG electrodes. This pattern is also seen for the alpha band. The pattern of significance suggests that within-gyrus correlations are highest, followed by within-lobe correlations, and finally acrosslobe correlations on gyri. The pattern was not statistically significant for the beta to high gamma band. We surmise that this is due to the already-low correlations that are observed at the shortest observable distance of $4 \mathrm{~mm}$ in these bands. We postulate that if the density of the grid were high enough, the same pattern would be observed in higher frequency bands as in the theta band; within-gyrus correlations are highest, followed by within-lobe correlations, and finally across-sulcus correlations.

\section{Discussion}

This study was done to empirically define the spatial spread function of ECoG signals towards understanding the relationship of neural frequency with spatial spread of activity across electrodes. We quantitatively defined the relationship of neural signal correlation and spatial distance. We further quantified how frequency band components affect this relationship, as higher frequencies have higher spatial resolution than low frequencies. We compared the spatial spread function across patients and across functional gyri, and noted consistency in the spatial fall off across patients and across functionally distinct areas. We then examined the effect of gyral and sulcal structural anatomy on spatial correlations.

There are three major contributions to the observed positive spatial correlations, not including artifact or a common reference. First, there are correlations that are due simply to the physical properties of the measured electric field; this is referred to as volume conduction. The magnitude of electric fields decrease as a function of one over distance squared (Cybulski et al 2014). Second, there are functional correlations in neural networks due to synaptic connectivity. These functional correlations could appear in regions far away from each other because networks are not constrained to neighboring areas. Third, there are correlations due to the properties of the underlying neurophysiological generators of electric signals. While the neural origins of the various canonical frequency bands are still a topic of debate, one theory is that low frequency signals are due to the spatial summation of synchronous dendritic inputs and that high frequency signals are the fields produced by spiking sub-populations and fast ionic and synaptic currents (Edwards et al 2009, 2010, Pesaran 2009, Lempka et al 2011). If this is the case, low frequency correlations are more widespread than high frequency correlations because their generators act as a synchronous unit over a larger area than a high frequency spiking subpopulation. All three major categories of correlation are presumably represented in the dataset that we have analyzed in this paper.

To date, the closest study to the one presented here was a spatial correlation analysis of ECoG signals across different electrode types in various places on the human cortex (Kellis et al 2015). This was done using small microwire arrays in addition to large clinical arrays with $1 \mathrm{~cm}$ spacing. The findings in this paper suggest similar patterns in spatial correlation fall off with distance for both tested arrays, for raw data. However, the reported spatial correlation fall offs are shallower than we observe. This is probably due to recording while the patient was not engaged in a task to specifically 
elicit activity in the recorded area. In our own analysis of correlations during rest versus activity, we observed a similar shallow fall off curve during rest. Interestingly, high-frequency modulation, which is believed to represent the activity of spatially localized neural processes, shows a greater change in correlation for rest versus task conditions than low frequency data. This is consistent with the idea that spatially discrete high frequency generators are recruited for a task, while low frequency generators maintain a brain state or modulate attention (Engel and Fries 2010, Donner and Siegel 2011). Importantly, our finding of stronger falloff of correlations during activity suggests that higher electrode densities may add specificity to observed task-related signals. Since distinct patterns of evoked activity may be observed even at small distances, increased spatial resolution may improve decoding of task-related output. Determining the optimal spacing between electrodes would allow us to determine at what spatial scale neural signals are redundant for a given task.

It would seem that the optimal spacing of electrodes would simply be to use the smallest inter-electrode distance possible that avoids shunting. However, there are other factors to consider. First, as density increases, electrode size necessarily decreases to make space and indeed to avoid electrical shunting. Smaller electrodes may not be ideal for ECoG recordings because they measure integrated activity of a smaller body of neural generators, and they potentially lose sensitivity to deeper cortical sources (Wodlinger et al 2011). Furthermore, as electrodes become smaller their impedance increases, making the signal-to-noise ratio lower than similar electrodes of a larger scale. Second, higher channel counts mean more wires, connectors, amplifiers, and data storage units. This is not trivial as channel counts increase into the thousands, though it may not be necessary if a more efficient use of materials can give a similar picture of the neural activity.

Considering the above as motivation, it is clear that determining the falloff of signal correlations over cortical distance has implications for the design of ECoG grids. Freeman et al performed a study on the optimal spacing of electrodes in ECoG for signals recorded from the human STG and motor cortex (Freeman et al 2000). The study was done with a linear microwire array, and it examined the spatial frequency of neural oscillations up to $50 \mathrm{~Hz}$. The conclusion was that for these frequency bands, the smallest spatial frequency observed above the noise floor was one that corresponds to $2.5 \mathrm{~mm}$ per cyclic spatial change. This correlates well with our shared variability observation for low frequency neural signals. Another more recent study (Slutzky et al 2010), used modeling of neural dipoles to estimate the optimal spacing of surface electrodes for human cortex. The resulting recommendation for electrode spacing was $1.4 \mathrm{~mm}$ correcting for Nyquist sampling, a measurement that again corresponds roughly to our observed and extrapolated correlations for low frequency neural data in the theta and alpha bands.

We have demonstrated the relationship between neural frequency and the spatial spread of signals at the cortical surface. Whether the application is during rest or active behavior will change the expected signal correlation over distance. The phase and amplitude observations have similar dependencies; however, phase correlations decrease more quickly with distance. This is of particular importance in the theta and alpha bands where phase is a common signal of interest.

Although this study included areas that were both sensorimotor and higher-order processing, correlations were computed for speech-related tasks in speech-related areas. Cortical encoding and tuning might be at different scales in other brain regions or even for other types of stimuli. A potential limitation of the fitting approach is that it leans toward the average of many data points, and therefore the resulting fit can over- or underestimate the activity spread from any one specific point. The results are an estimated fit averaged over several patients; as such, the cortical resolution suggested by these results should be interpreted as an average, not an absolute minimum. By the same token, taking the interquartile range leaves out the most highly correlated electrodes at each distance and can therefore be considered a conservative measure of shared signal.

Furthermore, our results provide novel information about the spatial spread of local field potential signals at the cortical surface. With ECoG grids of sufficient density and area, analysis of cortical activity can better describe the dynamics and functional connectivity of the cortex. Future studies can reveal if there are truly separable functional areas that respond to similar stimuli. The identification of functional areas corresponding to frequency of neural signal can shed light onto the origin of neural oscillations of various frequencies, most of which are not currently well understood.

\section{Conclusions}

The optimal spacing of ECoG electrodes depends heavily on the neural frequency band. The shape of the curve that defines this relationship is consistent across patients, but differs depending on which frequency band is represented and on which feature, amplitude or phase, is being represented. The dependence of this correlation falloff function on anatomical region is minimal, but falloff is stronger during speech-related tasks compared to rest. Correlations within functionally defined gyri are higher than those across sulci for low frequency oscillations.

\section{Acknowledgments}

The authors would like to thank M Leonard, D Moses, C Tang, M Sjerps, M Baud, B Dichter, D Conant, G Anumanchipalli, L Frank, C Schreiner, D Lowenstein, and M Maharbiz for helpful discussion on this work. This work was supported by grants from the NIH and the National Institute on Deafness and Other Communication Disorders (R01 DC012379 04, to EFC, and a Ruth L Kirschstein Postdoctoral NRSA F32DC014192-01, to LSH), DARPA SUBNETS W911NF-14-2-0043, the William K. Bowes, Jr Foundation, 
the Shurl and Kay Curci Foundation, and the McKnight Foundation.

\section{References}

Blakely T, Miller K J, Rao R P N, Holmes M D and Ojemann J G 2008 Localization and classification of phonemes using high spatial resolution electrocorticography (ECoG) grids Annual Int. Conf. IEEE Engineering in Medicine and Biology Society. pp 4964-7

Bouchard K E, Mesgarani N, Johnson K and Chang E F 2013 Functional organization of human sensorimotor cortex for speech articulation Nature 495 327-32

Breshears J D et al 2011 Decoding motor signals from the pediatric cortex: implications for brain-computer interfaces in children Pediatrics 128 e160-8

Bruns A, Eckhorn R, Jokeit H and Ebner A 2000 Amplitude envelope correlation detects coupling among incoherent brain signals Neuroreport 11 1509-14

Buckner R L and Vincent J L 2007 Unrest at rest: default activity and spontaneous network correlations Neurolmage 37 1091-6

Buzsaki G and Draguhn A 2004 Neuronal oscillations in cortical networks Science 304 1926-9

Cohen M R and Kohn A 2011 Measuring and interpreting neuronal correlations Nat. Neurosci. 14 811-9

Crone N, Boatman D, Gordon B and Hao L 2001 Induced electrocorticographic gamma activity during auditory perception Clin. Neurophysiol. (doi:10.1016/S1388-2457(00) 00545-9)

Crone N E, Korzeniewska A and Franaszczuk P J 2011 Cortical gamma responses: searching high and low Int. J. Psychophysiol. 79 9-15

Cybulski T R, Glaser J I, Marblestone A H, Zamft B M, Boyden E S, Church G M and Kording K P 2014 Spatial information in large-scale neural recordings bioRxiv. Front. Comput. Neurosci. 2014172

Donner T H and Siegel M 2011 A framework for local cortical oscillation patterns Trends Cogn. Sci. 15 191-9

Edwards E, Nagarajan S S, Dalal S S, Canolty R T, Kirsch H E, Barbaro N M and Knight R T 2010 Spatiotemporal imaging of cortical activation during verb generation and picture naming NeuroImage 50 291-301

Edwards E, Soltani M, Kim W, Dalal S S, Nagarajan S S, Berger M S and Knight R T 2009 Comparison of timefrequency responses and the event-related potential to auditory speech stimuli in human cortex J. Neurophysiol. 102 377-86

Elston G N 2003 Cortex, cognition and the cell: new insights into the pyramidal neuron and prefrontal function Cerebral Cortex. 13 1124-38

Engel A K and Fries P 2010 Beta-band oscillations-signalling the status quo? Curr. Opin. Neurobiol. 20 156-65

Flinker A, Chang E F, Barbaro N M, Berger M S and Knight R T 2011 Sub-centimeter language organization in the human temporal lobe Brain Lang. 117 103-9

Freeman W J, Rogers L J, Holmes M D and Silbergeld D L 2000 Spatial spectral analysis of human electrocorticograms including the alpha and gamma bands J. Neurosci. Methods 95 $111-21$

Fries P, Nikolić D and Singer W 2007 The gamma cycle Trends Neurosci. 30 309-16

Fukushima M, Saunders R C, Leopold D A, Mishkin M and Averbeck B B 2012 Spontaneous high-gamma band activity reflects functional organization of auditory cortex in the awake macaque Neuron 74 899-910

Garofolo J 1993 TIMIT acoustic-phonetic continuous speech corpus LDC93S1
Kajikawa Y and Schroeder C E 2011 How local is the local field potential? Neuron $72847-58$

Katzner S, Nauhaus I, Benucci A, Bonin V, Ringach D L and Carandini M 2009 Local origin of field potentials in visual cortex Neuron 61 35-41

Kellis S, Miller K, Thomson K, Brown R, House P and Greger B 2010 Decoding spoken words using local field potentials recorded from the cortical surface J. Neural Eng. 7056007

Kellis S, Sorensen L, Darvas F, Sayres C, O'Neill K, Brown R, House P, Ojemann J and Greger B 2015 Multi-scale analysis of neural activity in humans: implications for micro-scale electrocorticography Clin. Neurophysiol. 1-11

Kent A R and Grill W M 2014 Analysis of deep brain stimulation electrode characteristics for neural recording J. Neural Eng. 11 046010

Khodagholy D, Gelinas J N, Thesen T, Doyle W, Devinsky O, Malliaras G G and Buzsaki G 2015 NeuroGrid: recording action potentials from the surface of the brain Nat. Neurosci. 18 310-5

Lachaux Jean-Philippe, Axmacher Nikolai, Mormann Florian, Halgren Eric and Crone Nathan E 2012 High-frequency neural activity and human cognition: Past, present and possible future of intracranial EEG research Progress in Neurobiology 98 (3) 279-301

Lempka S F, Johnson M D, Moffitt M A, Otto K J, Kipke D R and McIntyre C C 2011 Theoretical analysis of intracortical microelectrode recordings J. Neural Eng. 8045006

Leski S, Lindén H, Tetzlaff T, Pettersen K H and Einevoll G T 2013 Frequency dependence of signal power and spatial reach of the local field potential PLoS Comput. Biol. 9 1-23

Leuthardt E C, Freudenberg Z, Bundy D and Roland J 2009 Microscale recording from human motor cortex: implications for minimally invasive electrocorticographic brain-computer interfaces Neurosurg. Focus 27 E10

Miller K J, Leuthardt E C, Schalk G, Rao R P N, Anderson N R, Moran D W, Miller J W and Ojemann J G 2007 Spectral changes in cortical surface potentials during motor movement J. Neurosci. 27 2424-32

Pesaran B 2009 Uncovering the mysterious origins of local field potentials Neuron 61 1-2

Pesaran B 2010 Neural correlations, decisions, and actions Curr. Opin. Neurobiol. 20 166-71

Ray S, Crone N E, Niebur E, Franaszczuk P J and Hsiao S S 2008 Neural correlates of high-gamma oscillations $(60-200 \mathrm{~Hz})$ in macaque local field potentials and their potential implications in electrocorticography J. Neurosci. 28 11526-36

Slutzky M W, Jordan L R, Krieg T, Chen M, Mogul D J and Miller L E 2010 Optimal spacing of surface electrode arrays for brain-machine interface applications J. Neural Eng. 7 26004

Viventi J et al 2011 Flexible, foldable, actively multiplexed, highdensity electrode array for mapping brain activity in vivo Nat. Neurosci. 14 1599-605

Wang W et al 2009 Human motor cortical activity recorded with Micro-ECoG electrodes, during individual finger movements Annual Int. Conf. IEEE Engineering in Medicine and Biology Society pp 586-9

Wodlinger B, Degenhart A D, Collinger J L, Tyler-Kabara E C and Wang W 2011 The impact of electrode characteristics on electrocorticography (ECoG) Proc. Annual Int. Conf. IEEE Engineering in Medicine and Biology Society, EMBS pp 3083-6

Womelsdorf Thilo and Fries Pascal 2007 The role of neuronal synchronization in selective attention Curr. Opin. Neurobiol. 17 154-60

Wrench A 1999 The MOCHA-TIMIT articulatory database

Xing D, Yeh C-I and Shapley R M 2009 Spatial spread of the local field potential and its laminar variation in visual cortex J. Neurosci. 29 11540-9 